\title{
Genetic relationship between olfactory response and fitness in Cotesia glomerata (L.)
}

\author{
Q Wang, H Gu and S Dorn \\ Institute of Plant Sciences/Applied Entomology, Swiss Federal Institute of Technology (ETH), Zurich, Switzerland
}

\begin{abstract}
The flight response of the parasitic wasp Cotesia glomerata (L.) to semiochemicals from a plant-host complex is subject to genetic variation. The significance of additive genetic variance for the odour-guided behaviour has been demonstrated by bidirectional selection. In order to understand the potential and constraints for phenotypic evolution in olfactory response under the pressure of natural selection, this study was to investigate genetic covariation between the odourguided behaviour and life-history traits and its genetic correlation with the efficiency of parasitism. A paternal halfsib analysis revealed that there was no significant genetic correlation between this behavioural character and any of three life-history traits examined (the development time of immature stages, the body size of female wasps, the number
\end{abstract}

of female wasps per brood). Comparisons between the selected high and low olfactory-response strains showed the lack of correlated responses in these life-history traits to bidirectional selection on the odour-guided behaviour. On the other hand, genotypic differences in the ability of olfactory response significantly affected the efficiency of parasitism. In comparison with the low olfactory-response strain, female wasps from the high olfactory-response strain were able to parasitize more host larvae in a wider area of habitats. This study provides the first evidence of links between olfactory response and population success in parasitoids from a genetic perspective.

Heredity (2004) 92, 579-584. doi:10.1038/sj.hdy.6800464

Keywords: parasitoid; Cotesia glomerata; olfactory response; life-history traits; parasitism; genetic covariation

\section{Introduction}

Parasitic wasps (parasitoids) rely to a larger degree on chemical signals in search for hosts in the field, although visual, acoustic and tactile stimuli are also involved in the host-location process (Vet and Dicke, 1992; Tumlinson et al, 1993). The volatiles emitted from the plants infested by host herbivores represent the most prevalent form of information about the presence of herbivore hosts, as such semiochemicals can be conveyed through air over distances (Vet, 1999). Since olfactory response plays an essential role in the survival and reproduction of individuals and hence population success in parasitoids, it will be subject to natural selection (Vet, 2001).

Empirical studies have shown that a significant proportion of phenotypic variation in odour-guided behaviours of parasitoids is genetic in origin (Prevost and Lewis, 1990; Gu and Dorn, 2000; Campan et al, 2002). Additive genetic variance in flight response to semiochemicals from a plant-host complex has been demonstrated directly by laboratory selection (Wang et al, 2003). The existence of additive genetic variance in a population predicts the potential of a character to respond to selection (Falconer, 1989). However, single characters will evolve together if they are correlated genetically with one another and either or both are correlated genetically with fitness (Stearns, 1976; Bell, 1997). Therefore, the knowledge of not only genetic variation for this

Correspondence: H Gu, Institute of Plant Sciences/Applied Entomology, Swiss Federal Institute of Technology (ETH), Clausiusstrasse 25/NW, CH-8092, Zurich, Switzerland. E-mail: hainan.gu@ipw.agrl.ethz.ch

Received 24 October 2003; accepted 19 February 2004 behavioural character but also the genetic covariation with other fitness traits is essential to address the issue of evolutionary changes in the olfactory response of parasitoids to the environment.

This study was to address two questions: (1) is olfactory response genetically correlated with other fitness components in parasitoids? (2) do genotypic differences in the ability of olfactory response influence the efficiency of parasitism? For these purposes, a sib analysis was conducted to investigate genetic correlations between flight response to semiochemicals from a plant-host complex and three life-history traits (the development time of immature stages, the body size of female wasps, and the number of female wasps per brood) in the parasitic wasp Cotesia glomerata (L.). Correlated responses in these life-history traits to bidirectional selection on flight response to semiochemicals from a plant-host complex were also examined by multigenerational comparisons of the two strains that were genetically differentiated in the odour-guided behaviour (Wang et al, 2003). Furthermore, the two selected strains were evaluated for their habitat colonization capability and host location efficiency.

\section{Materials and methods}

\section{Sib analysis}

Parasitoids originated from a Swiss field population of $C$. glomerata. The population samples were obtained from the host larvae (Pieris brassicae) parasitized by field wasps. In total, 52 potted cabbage plants (Brassica oleracea var. capitata cv. Mezzo) were distributed in cabbage fields at Feldi in Canton Zurich. Each of these potted plants 
carried 5-15 neonate larvae of $P$. brassicae. After exposure to field wasps for 2 days, these plants were brought back to an insectary. The recovered $P$. brassicae larvae were fed with cabbage leaves in insect cages $(30 \times 30 \times 30 \mathrm{~cm})$ within a climate chamber at $25^{\circ} \mathrm{C}, 70 \% \mathrm{RH}$ and L16:D8. Cocoon clusters of $C$. glomerata from the parasitized hosts were collected and kept at $21^{\circ} \mathrm{C}, 70 \% \mathrm{RH}$ and L16:D8.

Six cocoons were separated from each of the 97 fieldderived broods, and each of these cocoons was placed singly in a small vial for emergence. From the wasps obtained from each brood, either one male or one female was randomly chosen. The selected individuals were mixed as a group according to sex. Male and female groups were reared separately in nylon gauze cages $(30 \times 30 \times 30 \mathrm{~cm})$ and fed with honey and water. Three days later, 12 male and 60 female wasps were randomly taken from the respective group. Each male (sire) was allowed to mate with five females (dams). The females were placed one after another together with the male in a small transparent vial $(4 \times 2 \mathrm{~cm})$ that allowed direct observation of the mating process. A female was considered as having successfully mated only if copulation lasted for longer than $5 \mathrm{~s}$. After copulation, the mated female wasp was removed from the vial and transferred to a cylinder cage $(12.5 \times 16 \mathrm{~cm})$. The five dams (females) mated to the same sire (male) were kept together in a cylinder cage with honey and water.

Five 2nd-instar P. brassicae larvae were offered to each mated female wasp for parasitism 1 day after copulation. To avoid superparasitism (Gu et al, 2003), only one oviposition was allowed in each caterpillar. One host caterpillar was placed in a glass tube that held a female wasp, and immediately removed from the tube after completion of the first oviposition. The five $P$. brassicae parasitized by the same female wasp were reared together on cabbage leaves in a plastic box $(20 \times 10 \times 8.5 \mathrm{~cm})$. The duration of development before egression of parasitoid offspring was observed for each brood.

Two days after egression and pupation of parasitoids, the cocoon clusters (broods) were collected and placed separately in vials $(3 \times 5.5 \mathrm{~cm})$ according to hosts, with one cluster per vial. Emergence of each brood was checked for the number of male and female wasps. Wasps were fed with honey and water in a cylinder cage $(12.5 \times 16 \mathrm{~cm})$, but deprived of any contact with cabbageor host-related cues. Four to five days after emergence, 30 female wasps were randomly taken from different broods produced by a single dam, and tested for flight response to hexane extract from a plant-host complex in a wind tunnel, as described in Wang et al (2003). The flight test was conducted at a temperature of $20 \pm 1{ }^{\circ} \mathrm{C}$, relative humidity of $50-60 \%$, wind speed of $30-35 \mathrm{~cm} / \mathrm{s}$ and light intensity at 640-650 lux. Each individual was given a chance to take off from a release platform. After release, the wasp was observed for its behaviour within a maximum of $2 \mathrm{~min}$. A straightforward or zigzagging flight towards an odour source, which was set up upwind at a distance of $50 \mathrm{~cm}$ from the release platform, was recorded as 'flight response'. A random flying performance was considered failure in flight response to the odour source. The bioassay was conducted during 3 consecutive days. The same number of individuals from each dam was tested on each day as the wasps became available. Random samples were also taken from the broods derived from each dam to check the body size of female wasps. The body size of wasps was measured as the mean tibia length of the hind legs.

In total, 11 of the 12 sires used in the experiment had at least two dams that produced enough female offspring for the bioassay. The parasitoid offspring of each dam constituted a full-sib family and those from the different dams sharing the same sire comprised half-sib families. The percentage of flight response was calculated for each family since the odour-guided behaviour is a binary character. Percentages of flight response were subject to arcsine-square-root transformation prior to the correlation analysis. Product-moment correlations between the odour-guided behaviour and each of the three lifehistory traits (the development time of immature stages, the body size of female wasps, and the number of female wasps per brood) were calculated based on the mean values of paternal half-sib families, as such estimates are not confounded with the nonadditive genetic components such as dominance and maternal effects (Falconer, 1989). All analyses were performed with the statistical program StatView 5.0.1.

\section{Examination of correlated responses in life-history traits} Bidirectional selection led to significant divergence in flight response to semiochemicals from the plant-host complex between the two selected strains (Wang et al, 2003). Correlated responses to selection on the odourguided behaviour in three life-history traits (the development time of immature stages, the body size of female wasps and the number of female wasps per brood) were examined for the high and low olfactory-response strains in each generation of selection. As in the sib analysis, the development time of immature stages was recorded as the time from oviposition to egression from the host. The body size of female wasps was measured as the mean length of the two hind tibiae, as described above. The number of female wasps per brood was obtained by counting the wasps from a single host. To test for differences between the two strains, an analysis of variance (ANOVA) with repeated measures (ie the generation) was conducted using the program StatView.

\section{Evaluation of the efficiency of parasitism for the two selected strains}

The efficiency of parasitism for the two selected strains was evaluated in a greenhouse experiment. After four generations of selection, the progeny of the high and low olfactory-response strains were used for this experiment. Releases were carried out in neighbouring greenhouse compartments with the same environmental conditions: $24 \pm 3^{\circ} \mathrm{C}, 60 \% \mathrm{RH}$ and L16:D8. A cabbage plant patch with four rows of 10 plants was established in each compartment, using 2-month old potted plants ( $B$. oleracea L. var. capitata cv. Mezzo). At $24 \mathrm{~h}$ before wasp release, 12 plants, each infested by five 2 nd instar $P$. brassicae caterpillars, were randomly arranged among the 28 uninfested plants within the patch. Plants were positioned $10 \mathrm{~cm}$ apart from each other. In one experimental set-up, wasps from each strain were released in the centre of the plant patch, and in another set-up, they were released at a distance of $80 \mathrm{~cm}$ away from the cabbage patch. In each scenario, the two strains were 
released simultaneously, each in a separate compartment, and the release was replicated by changing compartments.

From each strain, 20 mated female wasps, aged 3-5 days, were randomly selected for each experimental replicate. They were released at 10:00 am. Every $3 \mathrm{~h}$ thereafter, four caterpillar-infested cabbage plants were randomly taken from the plant patch and replaced by uninfested plants. The distance of each sampled plant from the wasp-releasing point was noted. Sampling was made 3, 6 and $9 \mathrm{~h}$ after wasp release, respectively. To determine parasitism, all caterpillars on the same plant were reared within a single box $(20 \times 10 \times 8.5 \mathrm{~cm})$ in an insectary at $21 \pm 1{ }^{\circ} \mathrm{C}, 40-60 \% \mathrm{RH}$ and L16:D8 until pupation of the parasitoids. The product of parasitism rates on individual host-infested plants and their distances to the wasp release point was used to define the efficiency of parasitism. Thus, this efficiency index measures the capability of both habitat colonisation and host location. To test for differences in the efficiency of parasitism between the two selected strains, an ANOVA with repeated measures (ie the sampling time) was conducted for each release scenario, using the program StatView.

\section{Results}

\section{Paternal half-sib analysis for genetic correlations}

The odour-guided behaviour was positively correlated with the development time of immature stages and the number of females per brood and negatively with the tibia length of female wasps (Table 1). However, none of these correlation coefficients differed significantly from

Table 1 Genetic correlations between olfactory response and lifehistory traits (development time of immature stages, tibia length of female wasps, number of females per brood), based on mean values of paternal half-sib families

\begin{tabular}{lccc}
\hline Life-history trait & Correlation coefficient & $n$ & $P$-value \\
\hline Development time & 0.191 & 11 & 0.584 \\
Female tibia length & -0.219 & 11 & 0.530 \\
Females per brood & 0.402 & 11 & 0.229 \\
\hline
\end{tabular}

zero. Thus, there were no significant genetic correlations between olfactory response and these life-history traits in C. glomerata.

Examination of correlated responses in life-history traits After the first generation of selection on flight response to semiochemicals from the plant-host complex, the upward-selected strain already differentiated significantly from the downward-selected strain in the odourguided behaviour, and their divergence continued with further selection in subsequent generations. The data were shown in a previous paper (Wang et al, 2003). Changes in the three life-history traits also occurred during the four generations of selection (Table 2). The mean development time of immature stages in both strains decreased from the first to fourth generation of selection. The mean body size of female wasps, measured as the mean length of the two hind tibiae, increased from the first to the third generation, and then remained unchanged in the fourth generation. The mean number of females per brood increased from the first to the second generation of selection and then decreased in the third and fourth generations. However, these changes were not caused by selection on the odour-guided behaviour, as generational differences in none of these life-history traits were significant between the two strains, though the development time of immature stages and females per brood showed interactions between strain and the generation of selection (Table 3 ).

\section{Evaluation of the efficiency of parasitism for the two selected strains}

The two strains differed in the host-foraging activity of female wasps with respect to time elapse after release. When released in the middle of a cabbage plant patch, wasps from both strains parasitized host caterpillars close to the release point within the first $3 \mathrm{~h}$. As time elapsed, the patch area colonized and the number of hosts parasitized by female wasps from both strains increased. In comparison to the low olfactory-response strain, however, female wasps from the high olfactoryresponse strain parasitized more hosts in larger areas with processing time (Figure 1). In the case where wasps were released outside a plant patch, differences in the performance of the two strains were also evident. At $3 \mathrm{~h}$

Table 2 Mean values and standard deviations of life-history traits (development time of immature stages, body size of female wasps, number of females per brood) in the high and low olfactory-response strains of Cotesia glomerata with regard to different generations of selection (sample size shown in parentheses)

\begin{tabular}{|c|c|c|c|c|}
\hline \multirow[t]{2}{*}{ Generation of selection } & \multirow[t]{2}{*}{ Strain } & \multicolumn{3}{|c|}{ Life-history trait } \\
\hline & & Development time (days) & Female tibia length ( $\mathrm{mm})$ & Females per brood \\
\hline \multirow[t]{2}{*}{1} & High & $13.83 \pm 1.49(76)$ & $0.787 \pm 0.079$ & $16.4 \pm 14.1(42)$ \\
\hline & Low & $13.80 \pm 1.55(120)$ & $0.801 \pm 0.068$ & $17.5 \pm 15.8(48)$ \\
\hline \multirow[t]{2}{*}{2} & High & $13.15 \pm 0.39(93)$ & $0.824 \pm 0.067(250)$ & $19.8 \pm 15.4(80)$ \\
\hline & Low & $13.44 \pm 0.66(110)$ & $0.829 \pm 0.060$ & $20.9 \pm 16.8(69)$ \\
\hline \multirow[t]{2}{*}{3} & High & $12.42 \pm 0.76$ & $0.861 \pm 0.056$ & $13.2 \pm 11.0(87)$ \\
\hline & Low & $12.64 \pm 0.79(138)$ & $0.842 \pm 0.061$ & $16.9 \pm 16.6(70)$ \\
\hline \multirow[t]{2}{*}{4} & High & $11.96 \pm 0.82(115)$ & $0.842 \pm 0.061$ & $11.2 \pm 10.3(81)$ \\
\hline & Low & $11.84 \pm 0.91$ & $0.844 \pm 0.053$ & $6.5 \pm 9.5(89)$ \\
\hline
\end{tabular}


Table 3 Analysis of variance for differences in life-history traits (development time of immature stages, tibia length of female wasps, number of females per brood) between the high and low olfactory-response strains, using the general linear model with repeated measures (ie generations)

\begin{tabular}{|c|c|c|c|c|c|c|c|c|c|}
\hline \multirow[t]{3}{*}{ Source of variation } & \multicolumn{9}{|c|}{ Life-history traits } \\
\hline & \multicolumn{3}{|c|}{ Development time } & \multicolumn{3}{|c|}{ Female tibia length } & \multicolumn{3}{|c|}{ Females per brood } \\
\hline & $d f$ & $F$ & $\mathrm{P}$ & $d f$ & $F$ & $\mathrm{P}$ & $d f$ & $F$ & $\mathrm{P}$ \\
\hline Strain & 1 & 1.96 & $>0.05$ & 1 & 0.03 & $>0.05$ & 1 & 0.72 & $>0.05$ \\
\hline Generation & 3 & 137.42 & $<0.001$ & 3 & 59.52 & $<0.001$ & 3 & 16.09 & $<0.001$ \\
\hline Strain $\times$ generation & 3 & 2.72 & $<0.05$ & 3 & 0.68 & $>0.05$ & 3 & 3.31 & $<0.05$ \\
\hline
\end{tabular}

High olfactory-response strain Low olfactory-response strain
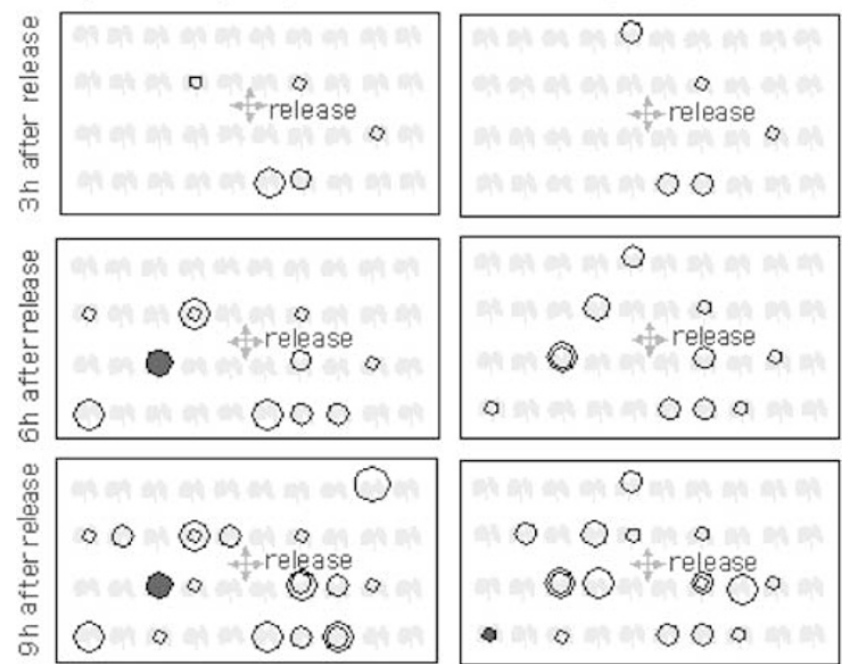

Figure 1 Temporal-spatial distribution of the host Pieris brassicae parasitized by the high and low olfactory-response strains of Cotesia glomerata, released in the centre of a cabbage plant patch. The data pooled from two replicates are presented since no significant differences were shown between replicates. The circle size indicates the number of parasitized host caterpillars $(1,2,3,4$ or 5$)$ on the plant ( $\odot$ - different levels of parasitism on a plant of the same position sampled in two replicates, $\bullet$ - the same level of parasitism on a plant of the same position sampled in two replicates).

after release, female wasps from the high olfactoryresponse strain had already parasitized some hosts whereas those from the low olfactory-response strain appeared to have not colonized the plant patch yet (Figure 2). In both replicates of release, the differences between the two strains continued as time elapsed.

The differences between the two strains are quantified using an index for the efficiency of parasitism (Figure 3). The analysis of variance showed that the efficiency of parasitism was significantly different between the two strains $(\mathrm{F}=7.60, \mathrm{df}=1,12, P<0.02)$ when wasps were released within plant patches, though strain $\times$ replicate interaction was also significant $(\mathrm{F}=5.61, \mathrm{df}=1,12$, $P<0.05)$. Despite clear differences between the two strains during the first $3 \mathrm{~h}$ after wasps were released outside plant patches, the mean values for the efficiency of parasitism were overlapped between the two strains after 6 and $9 \mathrm{~h}$ of release; thus their general efficiencies of parasitism were not statistically significant $(\mathrm{F}=2.46$, $\mathrm{df}=1,12, P>0.05)$. In both release scenarios, no significant differences were observed between replicates of release $(\mathrm{F}=0.16, \mathrm{df}=1,12, P>0.6$ for release within
High olfactory-response strain Low olfactory-response strain
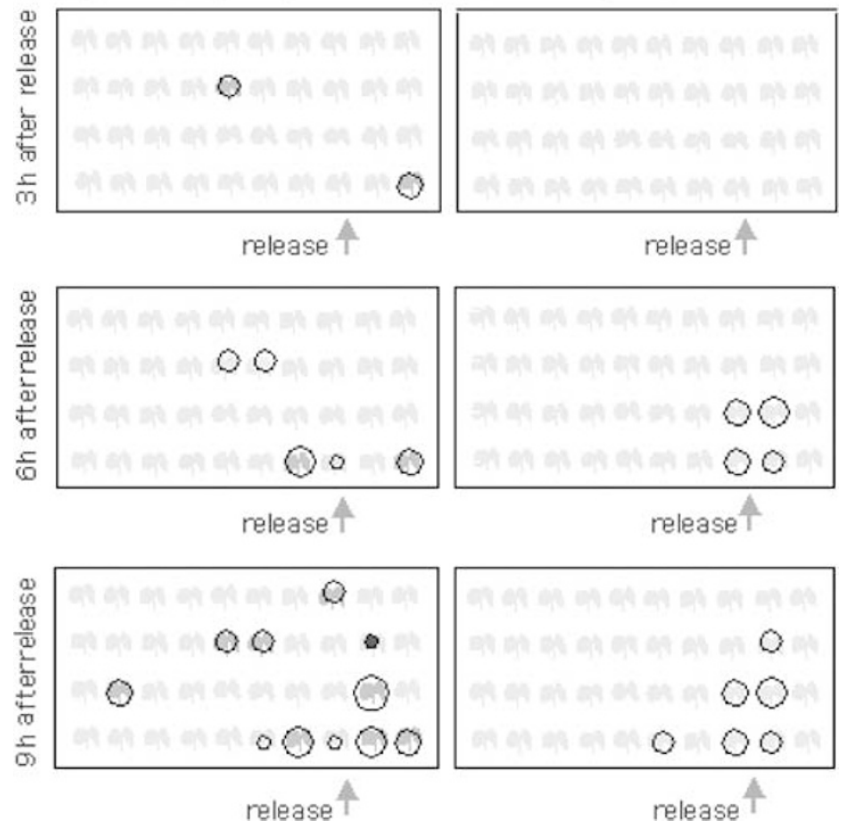

Figure 2 Temporal-spatial distribution of the host Pieris brassicae parasitized by the high and low olfactory-response strains of Cotesia glomerata, released outside a cabbage plant patch. The data pooled from two replicates are presented since no significant differences were shown between replicates. The circle size indicates the number of parasitized host caterpillars $(1,2,3,4$ or 5$)$ on the plant $(\bullet-$ the same level of parasitism on a plant of the same position sampled in two replicates).

plant patches; $\mathrm{F}=2.82, \mathrm{df}=1,12, P>0.1$ for release outside plant patches).

\section{Discussion}

The paternal half-sib analysis revealed no significant correlation between the odour-guided behaviour and the development time of immature stages, the body size of adults or the number of female wasps per host in $C$. glomerata. It should be pointed out, however, that the structure of genetic covariation among different characters could vary with the environmental conditions under which the experiment was carried out or due to sampling errors (Service and Rose, 1985). This is exemplified in a quantitative genetic study with the light brown apple moth, Epiphyas postvittana (Walker) (Lepidoptera: Tortricidae). In this herbivore insect, the structure of genetic covariations between flight capacity 

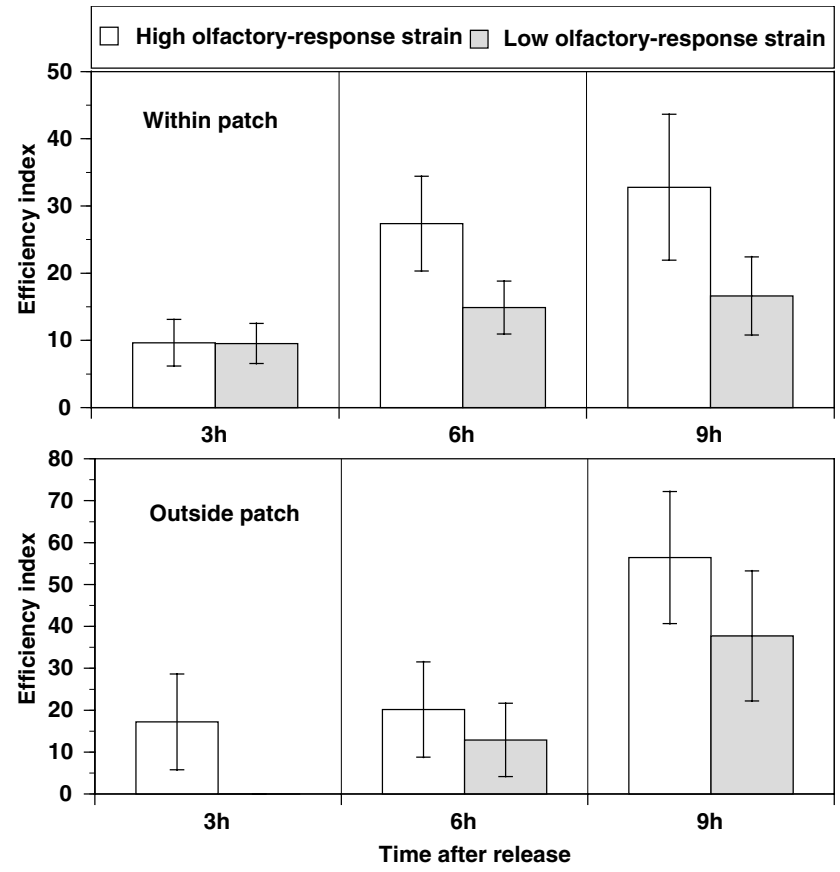

Figure 3 The efficiency of parasitism of the high and low olfactoryresponse strains of Cotesia glomerata (the upper part presents the data from the experimental scenario where female wasps were released in the centre of a cabbage plant patch, and the lower part the data from the experimental scenario where wasps were released outside a cabbage plant patch).

and life-history traits (Gu and Danthanarayana, 1992) and among life-history traits ( $\mathrm{Gu}$ and Danthanarayana, 2000) were shown to differ at two laboratory temperatures. The present experiment was carried out under constant laboratory conditions, which are different from the environmental conditions that $C$. glomerata experience in the field. In view of the possible environmental effect, caution must be taken when interpreting genetic correlations estimated from laboratory analyses and when extrapolating these results to different situations.

In comparison to sib analysis, selection experiments are considered to be more powerful tools to probe into the genetic architecture of organisms (Scheiner, 2002). Bidirectional selection on flight response to semiochemicals from the plant-host complex in C. glomerata diverged the two selected strains in opposite directions, demonstrating the presence of additive genetic variance in the odour-guided behaviour (Wang et al, 2003). However, the two selected strains did not show consistent differences in the life-history traits examined in different generations of selection. The lack of correlated responses in these life-history traits validated the results derived from the sib analysis. Therefore, there is no evidence for genetic covariation between the odourguided behaviour and life-history traits in this parasitoid species. The absence of significant genetic covariation between the odour-guided behaviour and other fitness traits supports that the ability of flight response to the herbivore-induced plant odour is located at the sensory level rather than in flight capacity (Wang et al, 2003). Although the empirical data obtained from this study do not warrant a generalization that olfactory response is not correlated with life-history traits in parasitoids, a genetic analysis for a natural population of Drosophila
Covariation between olfactory response and fitness

$Q$ Wang et al

melanogaster also showed that genetic correlations between an odour-guided behaviour (ie benzaldehyde avoidance) and fitness traits were not significantly different from zero (Mackay et al, 1996). It has been interpreted that the odour-guided behaviour shows low significant genetic correlations with fitness traits if the number of loci affecting olfactory response is low compared to the number of loci affecting total fitness (Mackay et al, 1996). Whether this interpretation applies to the case of $C$. glomerata remains to be answered.

The odour-guided behaviour directly influenced the efficiency of parasitism in C. glomerata. The strain with high olfactory response was more capable of habitat exploitation and host location than the strain with low olfactory response. Clearly, these differences resulted directly from genetic differentiation in the odour-guided behaviour, since the two strains were not significantly different in life-history traits, including the body size of female wasps (this study) and flight capacity (Wang et al, 2003). Apparently, the positive relation between olfactory response and host-foraging performance in parasitoids is in contrast to the relationship between olfactory response and prey-searching activities in predators. In the predatory mite, Phytoseiulus persimilis (Athias-Henriot), the strain with strong olfactory response tended to have a shorter residence time on infested leaf disks, leading to less efficacy in exploiting a local prey patch in comparison to the strain with weak olfactory response (Margolies et al, 1997). Nevertheless, the apparent trade-off between patch location and patch residency in predatory mites was compounded by phenotypic correlation between patch residency and prey consumption (Jia et al, 2002).

Previous studies have demonstrated that there is significant additive genetic variance for olfactory response to semiochemicals in the natural populations of C. glomerata (Gu and Dorn, 2000; Wang et al, 2003). In theory, the existence of genetic variance in a character can be due to the antagonistic pleiotropy manifested as negative genetic correlations between the character in question and fitness (Rose and Charlesworth, 1981; Rose, 1982) and/or result from disruptive selection in a variable environment through genotype-environment interaction (Ewing, 1979; Gillespie and Turelli, 1989). Comparisons of the two selected strains in three lifehistory traits and the efficiency of parasitism do not suggest that there are negative genetic correlations between the odour-guided behaviour and other fitness traits in this parasitoid. These findings imply that the habitat heterogeneity of this generalist parasitoid might play a major role in the maintenance of genetic variance in the odour-guided behaviour within natural populations, as has been suggested previously (Wang et al, 2003). Since the life-history traits, such as the development time of immature stages and the number of females per brood, in different olfactory response strains varied differently with different generations of selection, such interaction could also contribute to the presence of genetic variance for olfactory response in the parasitoid populations.

In summary, the genotypes of $C$. glomerata that show a higher response to semiochemicals from the plant-host complex are more efficient in exploiting habitats and locating hosts than those with a lower olfactory response, though the life-history traits appeared not to be 
correlated with the odour-guided behaviour. This is the first evidence showing a genetic relationship between olfactory response and the efficiency of parasitism and hence with population success in parasitoids, although the link between olfactory response and the host-location capability was demonstrated by various studies on conditioning or associative learning in other parasitoids (Papaj and Vet, 1990; Turlings et al, 1993). Empirical studies on the subject in more species will advance our understanding of the tri-trophic interactions involving plants, herbivorous insects and parasitoids from a genetic prospective. Concurrently, the knowledge based on further empirical data may point to new possibilities of improving parasitoids as biological control agents by genetic manipulation of their behavioural response to herbivore-induced plant chemicals. In addition, the study of possible interaction with learning of hostfinding cues can precisely evaluate the advantages of improved olfactory response through genetic selection.

\section{Acknowledgements}

Thanks are due to Henry Wöhrnschimmel, David Wettstein, Lena Obrist and Tanja Christoffel for assistance with insect cultures. We are grateful to Dr Anja Rott, Dr Kathrin Tschudi-Rein and Dr Jörg Samietz for helpful comments on the manuscript. The comments and suggestions from an anonymous reviewer improved the manuscript.

\section{References}

Bell G (1997). Selection — The Mechanism of Evolution. Chapman \& Hall: London.

Campan E, Couty A, Carton Y, Phamdelègue MH, Kaiser L (2002). Variability and genetic components of innate fruit odour recognition in a parasitoid of Drosophila. Physiol Entomol 27: 243-250.

Ewing E (1979). Genetic variation in a heterogeneous environment. VII. Temporal and spatial heterogeneity in infinite populations. Am Nat 114: 197-212.

Falconer DS (1989). Introduction to Quantitative Genetics. 3rd edn. Longman: London.

Gillespie JH, Turelli M (1989). Genotype-environment interactions and the maintenance of polygenic variation. Genetics 121: $129-138$

$\mathrm{Gu} \mathrm{H}$, Danthanarayana W (1992). Quantitative genetic analysis of dispersal in Epiphyas postvittana. II. Genetic covariations between flight capacity and life-history traits. Heredity 68: 61-69.

Gu H, Danthanarayana W (2000). Genetic variation in the lifehistory traits of Epiphyas postvittana: population structure and local adaptation. Austral Ecol 25: 394-401.
$\mathrm{Gu} \mathrm{H}$, Dorn S (2000). Genetic variation in behavioral response to herbivore-infested plants in the parasitic wasp, Cotesia glomerata(L.) (Hymenoptera: Braconidae). J Insect Behav 13: 141-156.

Gu H, Wang Q, Dorn S (2003). Superparasitism in Cotesia glomerata: response of hosts and consequences for parasitoids. Ecol Entomol 28: 422-431.

Jia F, Margolies DC, Boyer JE, Charlton RE (2002). Genetic variation in foraging traits among inbred lines of a predatory mite. Heredity 89: 371-379.

Mackay TFC, Hackett JB, Lyman RF, Wayne ML, Anholt RH (1996). Quantitative genetic variation of odor-guided behavior in a natural population of Drosophila melanogaster. Genetics 144: 727-735.

Margolies DC, Sabelis MW, Boyer Jr JE (1997). Response of a phytoseiid predator to herbivore-induced plant volatiles: selection on attraction and effect on prey exploitation. J Insect Behav 10: 695-709.

Papaj DR, Vet LEM (1990). Odor learning and foraging successin the parasitoid Leptopilina heterotoma. J Chem Ecol 16: 3137-3150.

Prevost G, Lewis WJ (1990). Heritable differences in the response of the braconid wasp Microplitis croceipes to volatile allelochemicals. J Insect Behav 3: 277-287.

Rose MR (1982). Antagonistic pleiotropy, dominance, and genetic variation. Heredity 48: 63-78.

Rose MR, Charlesworth B (1981). Genetics of life history in Drosophila melanogaster. I. Sib analysis of adult females. Genetics 97: 173-186.

Service PM, Rose MR (1985). Genetic covariation among lifehistory components: the effect of novel environments. Evolution 39: 943-945.

Scheiner SM (2002). Selection experiments and the study of phenotypic plasticity. J Evol Biol 15: 889-898.

Stearns SC (1976). Life-history tactics: a review of the ideas. Q Rev Biol 51: 3-47.

Tumlinson JH, Turlings TCJ, Lewis WJ (1993). Semiochemically mediated foraging behavior in beneficial parasitic insects. Arch Insect Biochem Physiol 22: 385-391.

Turlings TCJ, Wäckers FL, Vet LEM, Lewis WJ, Tumlinson JH (1993). Learning of host-finding cues by hymenopterous parasitoids. In: Papaj DR, AC Lewis (eds), Insect Learning: Ecology and Evolutionary Perspectives. Chapman \& Hall: New York, pp 51-78.

Vet LEM (1999). From chemical to population ecology: infochemical use in an evolutionary context. J Chem Ecol 25: $31-49$.

Vet LEM (2001). Parasitoid searching efficiency links behaviour to population process. Appl Entomol Zool 36: 399-408.

Vet LEM, Dicke M (1992). Ecology of infochemical use by natural enemies in a tritrophic context. Annu Rev Entomol 37: 141-172.

Wang Q, Gu H, Dorn S (2003). Selection on olfactory response to semiochemicals from a plant-host complex in a parasitic wasp. Heredity 91: 430-435. 